\title{
Postoperative Irradiation for Rectal Cancer Increases the Risk of Small Bowel Obstruction After Surgery
}

\author{
Nancy N. Baxter, MD, PhD, *† Lacey K. Hartman, MPP, $\neq$ Joel E. Tepper, MD,\| \\ Rocco Ricciardi, MD, I Sara B. Durham, MSc, $\neq$ and Beth A. Virnig, PhD, MPH $\$$
}

\begin{abstract}
Objective: To determine the risk of small bowel obstruction (SBO) after irradiation (RT) for rectal cancer

Background: SBO is a frequent complication after standard resection of rectal cancer. Although the use of RT is increasing, the effect of RT on risk of SBO is unknown.

Methods: We conducted a retrospective cohort study using Surveillance, Epidemiology, and End Results cancer registry data linked to Medicare claims data to determine the effect of RT on risk of SBO. Patients 65 years of age and older diagnosed with nonmetastatic invasive rectal cancer treated with standard resection from 1986 through 1999 were included. We determined whether patients had undergone RT and evaluated the effect of RT and timing of RT on the incidence of admission to hospital for SBO, adjusting for potential confounders using a proportional hazards model.

Results: We identified a total of 5606 patients who met our selection criteria: 1994 (36\%) underwent RT, 74\% postoperatively. Patients were followed for a mean of 3.8 years. A total of 614 patients were admitted for SBO over the study period; $15 \%$ of patients in the RT group and $9 \%$ of patients in the nonirradiated group $(P<0.001)$. After controlling for age, sex, race, diagnosis year, type of surgery, and stage, we found that patients who underwent postoperative RT were at higher risk of SBO, hazard ratio 1.69 (95\% CI, 1.3-2.1). However, the long-term risk associated with preoperative irradiation
\end{abstract}

From the *Department of Surgery, St. Michael's Hospital, University of Toronto, Toronto, Ontario, Canada; $\dagger$ Department of Health Policy, Management, and Evaluation, University of Toronto, Toronto, Ontario, Canada; †Division of Health Services Research and Policy, University of Minnesota School of Public Health, Minneapolis, MN; §University of Minnesota Comprehensive Cancer Center, Minneapolis, MN; ||Department of Radiation Oncology and the UNC/Lineberger Comprehensive Cancer Center, University of North Carolina School of Medicine, Chapel

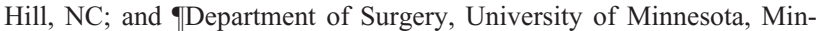
neapolis, $\mathrm{MN}$.

Supported by a Veterans of Foreign Wars Surgical Oncology research grant and by the University of Minnesota Comprehensive Cancer Center. Dr Baxter is supported by an American Society of Clinical Oncology Career Development Award and a Canadian Institutes of Health New Investigator Award.

The authors have no financial or personal relationships with other people or organizations that could bias this work. Dr. Baxter, as the corresponding author, has had full access to all the data in the study and has final responsibility for the decision to submit this manuscript for publication.

Reprints: Nancy Baxter, MD, PhD, Department of Surgery, St. Michael's Hospital, University of Toronto, 30 Bond Street, CC 16-040, Toronto, Ontario M5B 1W8, Canada. E-mail: baxtern@smh.toronto.on.ca.

Copyright (C) 2007 by Lippincott Williams \& Wilkins

ISSN: 0003-4932/07/24504-0553

DOI: $10.1097 / 01$. sla.0000250432.35369.65 was not statistically significant (hazard ratio, 0.89 ; 95\% CI, $0.55-$ 1.46).

Conclusions: Postoperative but not preoperative RT after standard resection of rectal cancer results in an increased risk of SBO over time.

(Ann Surg 2007;245: 553-559)

In 1990, the National Institutes of Health Consensus Conference established adjuvant postoperative radiation therapy, along with chemotherapy, as the standard of care for achieving regional control of stage II and III rectal cancer after surgical resection in the United States. ${ }^{1}$ Numerous studies have substantiated the benefits of adjuvant radiation therapy for preventing local recurrence in patients with rectal cancer $^{2-5}$; thus, radiation therapy is commonly used in the United States. In a previous population-based study using SEER data, we found that, in the year $2000,59 \%$ of patients with AJCC stage II rectal cancer and $68 \%$ with AJCC III rectal cancer underwent irradiation, ${ }^{6}$ and this rate was increasing substantially over time. With mounting evidence of the superiority of preoperative irradiation, ${ }^{7}$ the proportion of patients undergoing preoperative versus postoperative radiation therapy has also increased.

During radiation therapy, a significant amount of small bowel may lie in the irradiated field and receive a substantial radiation dose. Indeed, acute small bowel toxicity is one of the more common dose-limiting toxicities of irradiation for rectal cancer. ${ }^{8}$ Chronic gastrointestinal symptoms after treatment of rectal cancer are common ${ }^{9,10}$; however, in many cases, it is difficult to attribute problems to any specific aspect of care (surgery, chemotherapy, or irradiation). For example, small bowel obstruction (SBO) after major abdominal surgery is a frequent occurrence. ${ }^{11}$ Therefore, it may be difficult to determine if RT results in an increased risk above baseline. While there is some evidence that there may be less small bowel toxicity with preoperative irradiation, ${ }^{12}$ the data are primarily based on short-course irradiation (with limited generalizability to most North American centers) and without concurrent chemotherapy. We therefore designed this study to determine if irradiation is associated with an increased rate of SBO in patients with resectable rectal cancer, and to 
evaluate other factors (particularly type of surgery and timing of irradiation) that might influence the relationship.

\section{MATERIALS AND METHODS}

\section{Data}

We used Surveillance, Epidemiology, and End Results (SEER) cancer registry data linked to Medicare enrollment records and utilization data (SEER-Medicare). SEER, a population-based cancer registry sponsored by the National Cancer Institute, collects information on cancer incidence and survival from 11 population-based cancer registries; these 11 registries include about $14 \%$ of the U.S. population. ${ }^{13}$ The information collected by SEER includes patient characteristics, primary tumor site, tumor stage, first course of treatment (including surgery and irradiation), timing of irradiation, follow-up for vital status, and cause of death as recorded on the death certificate. ${ }^{13}$

Medicare provides comprehensive health care for about $98 \%$ of the U.S. population age 65 or older. Cancer cases reported to SEER have been matched to the Medicare master enrollment file, to facilitate population-based health services research. Medicare eligibility has been identified for $94 \%$ of people 65 or older identified by SEER. ${ }^{14}$ For Medicare enrollees who do not participate in a managed care plan, claims data for hospitalizations are available through the Medicare Provider Analysis and Review (MedPAR) file. SEER-Medicare includes cancer cases reported to SEER from 1973 through 1999 and all Medicare claims for people diagnosed with cancer from 1991 through 2001. Hospitalization data (per the MedPAR file) are available from 1986 through 2001. For this study, we evaluated only hospitalization for SBO and therefore were able to determine the rate of admission for SBO since 1986.

\section{Patients}

Included in our study were patients age 65 or older who were diagnosed with invasive, nonmetastatic rectal cancer from January 1, 1986, through December 31, 1999. We included only patients who underwent radical resection of rectal cancer (SEER site-specific surgery 30 through 90 ) for the first course of treatment.

Excluded from our study were patients with a previous cancer diagnosis and patients who developed a second cancer; patients with in situ, metastatic, or unstaged cancers; patients whose cancer was diagnosed by autopsy or first cited on the death certificate; patients who did not undergo surgery, or had local excision only; patients whose radiation status was unknown or who received only radioactive implants, radioisotopes, or other forms of radiation besides conventional therapy; patients who were enrolled in a managed care organization any time from 6 months prior to cancer diagnosis (because Medicare files do not include insurance claims data on managed care enrollees); patients whose race was unknown; and patients who presented with the diagnosis of SBO at the time of cancer diagnosis or within the month following diagnosis. Cause of death is available from SEER to December 31, 2000; therefore, this was considered the last date of potential follow-up for the purposes of this study.

\section{Analysis}

SEER routinely collects data on the first course of treatment, including radiation therapy. For our study, patients who underwent external-beam irradiation were defined as the irradiated group. The nonirradiated group was defined as patients who were prescribed no radiation therapy or refused such treatment. We also determined if irradiation was delivered before surgery (preoperative irradiation) or after surgery (postoperative irradiation). SBO was defined from the MedPAR data from 1986 through 2000 using the International Classification of Disease 9 (ICD-9) code $^{15} 560$ (intestinal obstruction without mention of hernia). For patients admitted with a diagnosis of SBO, we determined if further episodes developed over time, if surgery was performed during the admission, and assessed vital status at discharge for each episode.

We compared demographic variables between the irradiated and nonirradiated groups, using the $\chi^{2}$ test for categorical variables, the Student $t$ test for continuous normally distributed variables, and the Wilcoxon rank sum test for continuous variables that were not normally distributed. We calculated Kaplan-Meier curves representing the time from cancer diagnosis to SBO, and compared the curves using the Wilcoxon method. We compared the irradiated groups to the nonirradiated group and also compared the preoperative, postoperative, and nonirradiated groups. To control for potential confounders, we constructed a proportional hazards model to evaluate the relationship between irradiation and $\mathrm{SBO}$, adjusting for age at cancer diagnosis (in 5-year increments), sex, race (black vs. non-black), type of surgery (abdominoperineal resection [APR] vs. other radical surgery [non-APR]) stage of disease (local or regional stage), SEER registry, and time period of diagnosis (1986-1990, 19911995, and 1996-1999). We tested the model for possible interaction effects between irradiation and stage of disease, race, age, type of surgery, and time period of diagnosis. Follow-up was calculated as the time between diagnosis and admission for SBO. Censoring events included patient death, survival after 12 years of follow-up, and end of follow-up (December 31, 2000).

Because our study used preexisting data with no personal identifiers, the Human Subjects Committee of the University of Minnesota's Institutional Review Board determined that it was exempt from review. We performed the statistical analysis using SAS version 9.1. All statistical tests were 2 sided, and we considered a $P$ value of less than 0.05 to be statistically significant. In the past, we used similar methodology successfully to evaluate the effect of irradiation on the risk of pelvic fracture in older women with pelvic malignancies. ${ }^{16}$

\section{RESULTS}

A total of 5606 patients met our study's selection criteria. Patient characteristics are summarized in Table 1. Of these 5606 patients 1994 (35.6\%) underwent radiation therapy (irradiated group) and 3612 (64.4\%) did not (nonirradiated group). The majority of patients undergoing irradiation did so in a postoperative fashion $(73.6 \%)$. We could not 
TABLE 1. Patient Characteristics

\begin{tabular}{|c|c|c|c|c|c|}
\hline Variable & Category & All Patients & $\begin{array}{l}\text { Nonirradiated } \\
\text { Group }\end{array}$ & Irradiated Group & $P$ \\
\hline Total patients & (\% irradiated) & 5606 & $3612(64.4 \%)$ & $1994(35.6 \%)$ & NA \\
\hline \multirow[t]{3}{*}{ Age at diagnosis (yr) } & $65-69$ & $1313(47.1 \%)$ & $694(19.2 \%)$ & $619(31.0 \%)$ & $<0.0001$ \\
\hline & $75-79$ & $1313(34.5 \%)$ & $860(23.8 \%)$ & $453(22.7 \%)$ & \\
\hline & $80+$ & $1475(19.4 \%)$ & $1189(32.9 \%)$ & $286(14.3 \%)$ & \\
\hline \multirow{2}{*}{ Year at diagnosis } & 1991-1995 & $2072(36.6 \%)$ & $1313(36.4 \%)$ & $759(38.1 \%)$ & \\
\hline & $1996+$ & $1632(40.7 \%)$ & $968(26.8 \%)$ & $664(33.3 \%)$ & \\
\hline \multirow[t]{2}{*}{ Race } & Non-Black & $5.379(35.7 \%)$ & 3457 (95.7\%) & $1922(96.4 \%)$ & 0.22 \\
\hline & Black & $227(31.7 \%)$ & $155(4.3 \%)$ & $72(3.6 \%)$ & \\
\hline \multirow[t]{2}{*}{ Stage } & Localized & $2659(18.3 \%)$ & $2172(60.1 \%)$ & $487(24.4 \%)$ & $<0.0001$ \\
\hline & Regional & $2947(51.1 \%)$ & $1440(39.9 \%)$ & $1507(75.6 \%)$ & \\
\hline \multirow[t]{2}{*}{ Small bowel obstruction } & Yes & $614(48.5 \%)$ & $316(8.8 \%)$ & $298(14.9 \%)$ & $<0.0001$ \\
\hline & No & $4992(34.0 \%)$ & $3296(91.2 \%)$ & $1696(85.1 \%)$ & \\
\hline
\end{tabular}

NA indicates not applicable.

determine timing of irradiation in $102(5 \%)$ patients undergoing RT.

The average age at diagnosis was 75.2 years. Patients in the irradiated group were slightly younger (average, 73.2 years) than patients in the nonirradiated group (average, 76.4 years) $(P<0.001)$. Mean follow-up time for the entire cohort was 3.8 years. Mean follow-up time was slightly greater for the nonirradiated group (4.0 years) than the irradiated group (3.5 years) $(P<0.001)$, potentially because proportionately fewer patients were treated with RT in the early time period (28.6\% of patients treated with RT, 1986-1990) than in the later time period (33.3\% of patients, 1996-1999) $(P<$ $0.001)$, consistent with the known increase in the use of RT in the treatment of rectal cancer during the timeframe of the study. ${ }^{6}$ In addition, patients in the irradiated group were more likely to have been diagnosed with regional stage of disease $(75.6 \%)$ than patients in the nonirradiated group $(39.9 \%)$ $(P<0.001)$.

A total of 614 patients (11\%) were admitted with a primary diagnosis of SBO over the study period. The cumulative incidence of hospital admission for SBO was greater in the irradiated group than in the nonirradiated group (Fig. 1). The cumulative incidence of SBO at 5 years was $18 \%$ in the irradiated group versus $11 \%$ in the nonirradiated group. For those undergoing preoperative irradiation $(n=425)$, the cumulative risk of SBO at 5 years was $14 \%$ as compared with $20 \%$ in those undergoing postoperative irradiation. However, from the Kaplan-Meier curve (Fig. 2), the risk of SBO in the preoperative irradiation and postoperative irradiation groups was similar for the first 12 months; thereafter, the risk of SBO in the group undergoing preoperative irradiation was similar to the risk in patients not undergoing irradiation. Of note, patients undergoing preoperative irradiation had a mean fol- low-up of only 2.9 years (most patients undergoing preoperative irradiation were diagnosed after 1996).

We used a proportional hazards model to evaluate the influence of radiation therapy on incidence of admission for SBO (Table 2). After controlling for other factors, we found that patients undergoing irradiation had a higher risk of admission for SBO over time than patients who did not undergo irradiation (hazard ratio associated with irradiation = $1.53 ; 95 \%$ confidence interval, 1.28-1.83). Being black and having regional (vs. local) cancer also increased risk of SBO. However, we found no statistically significant interaction effects between these factors and radiation status on risk of admission for SBO over time. Type of surgery (APR vs. other radical resection) did not influence the risk of SBO.

Because there was evidence of violation of the proportional hazards assumption for patients undergoing preoperative irradiation (with a change in risk after 12 months) to evaluate the long-term effect of timing of irradiation, we repeated our proportional hazards model starting 12 months after diagnosis. To evaluate the short-term effect, we performed a logistic regression comparing the proportion of patients developing an SBO in the first year between the 3 groups. After adjusting for other covariates, within the first 12 months of diagnosis patients who underwent postoperative irradiation were statistically significantly more likely to have an admission for SBO than patients who did not undergo irradiation (odds ratio $=1.83 ; 95 \%$ CI, 1.30-2.58). Patients undergoing preoperative irradiation were also more likely to have an admission for SBO within the first 12 months of diagnosis than patients who did not undergo irradiation, although this did not achieve statistical significance $(\mathrm{OR}=$ 1.59; 95\% CI, 0.95-2.67). In our proportional hazards model, preoperative irradiation was not associated with an increased 


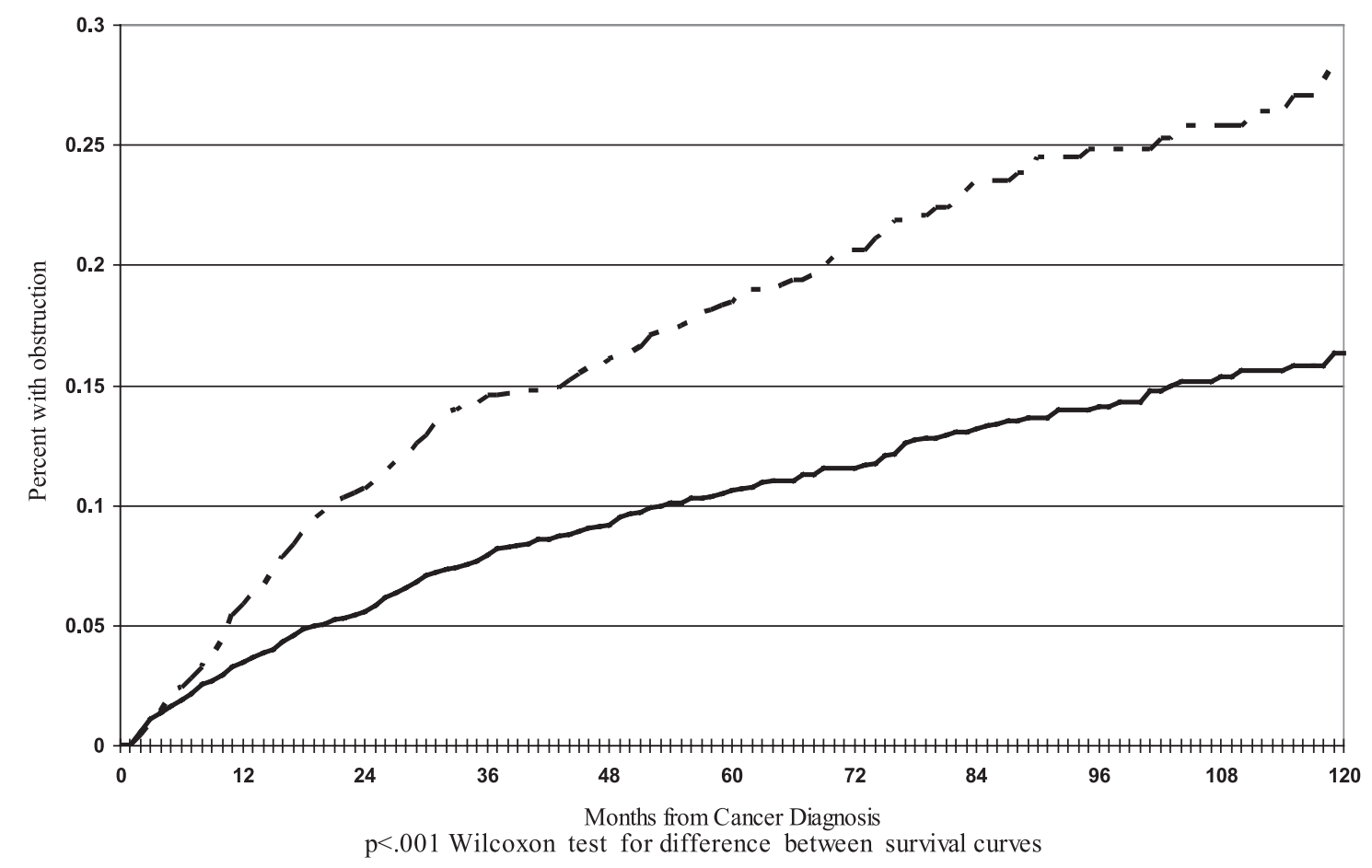

Number at Risk

$\begin{array}{lccc} & \text { Baseline } & 5 \text { years after diagnosis } & \text { 10 years after diagnosis } \\ \text { Irradiated group } & 1994 & 477 & 96 \\ \text { Nonirradiated group } & 3612 & 1152 & 311\end{array}$

FIGURE 1. Time from rectal cancer diagnosis to small bowel obstruction. Irradiated group is represented by the broken line; nonirradiated group, solid line.

risk of SBO more than 12 months after diagnosis $(\mathrm{HR}=$ 0.89; 95\% CI, 0.55-1.46) (Table 3). However, postoperative irradiation was associated with a statistically significant longterm risk of $\mathrm{SBO}(\mathrm{HR}=1.69 ; 95 \% \mathrm{CI}, 1.34-2.14)$.

Of the 578 patients who survived their first admission for SBO, 156 (25.4\% of patients admitted for SBO) were admitted for a second obstruction during the study period. In the irradiated group, 78 patients required at least 2 admissions for SBO (3.9\% of total cohort) versus 78 patients in the nonirradiated group $(2.2 \%$ of total cohort $)(P<0.001)$, although for the 578 patients surviving their first admission for SBO, irradiation did not increase the likelihood of recurrence $(27.8 \%$ of patients admitted for $\mathrm{SBO}$ in the irradiated group experienced recurrence vs. $26.3 \%$ of patients in the nonirradiated group, $P=0.71)$. A total of 249 patients required surgery for SBO during admission, $6.6 \%$ of all patients in the irradiated group and $3.2 \%$ of all patients in the nonirradiated group $(P<0.001)$. Of the 614 patients who had an admission for SBO in the study period, $8.1 \%(n=50)$ died during admission. There was no significant difference in rates of mortality from small bowel obstruction by irradiation status $(P=0.10)$.

Patients undergoing irradiation were more likely to have regional stage disease than those not undergoing irradi- ation and thus were at a higher risk of cancer recurrence. As peritoneal recurrence can present with bowel obstruction, we were concerned about the risk of residual confounding. We therefore repeated our analysis excluding all patients $(\mathrm{n}=$ 1754) who died of colorectal cancer in the study period and found no substantive differences (data not shown).

\section{DISCUSSION}

With greater awareness of the benefits of adjuvant irradiation for patients with rectal cancer, the number of rectal cancer patients treated in this fashion has dramatically increased. ${ }^{6}$ However, given that treatment is delivered with a curative intent, it is essential that long-term consequences for survivors are fully understood and, where possible, that factors modifying risk are identified. Our population-based, retrospective cohort study demonstrates that irradiation is associated with an increased risk of SBO for patients treated with radical resection of rectal cancer. Consistent with previous literature, ${ }^{17}$ we found that $\mathrm{SBO}$ is a frequent complication of surgery for rectal cancer; the risk of admission for SBO within 5 years of rectal cancer diagnosis was $11 \%$ in our surgery only group. However, because of the high baseline risk, the hazard ratio associated with irradiation (1.5) resulted 


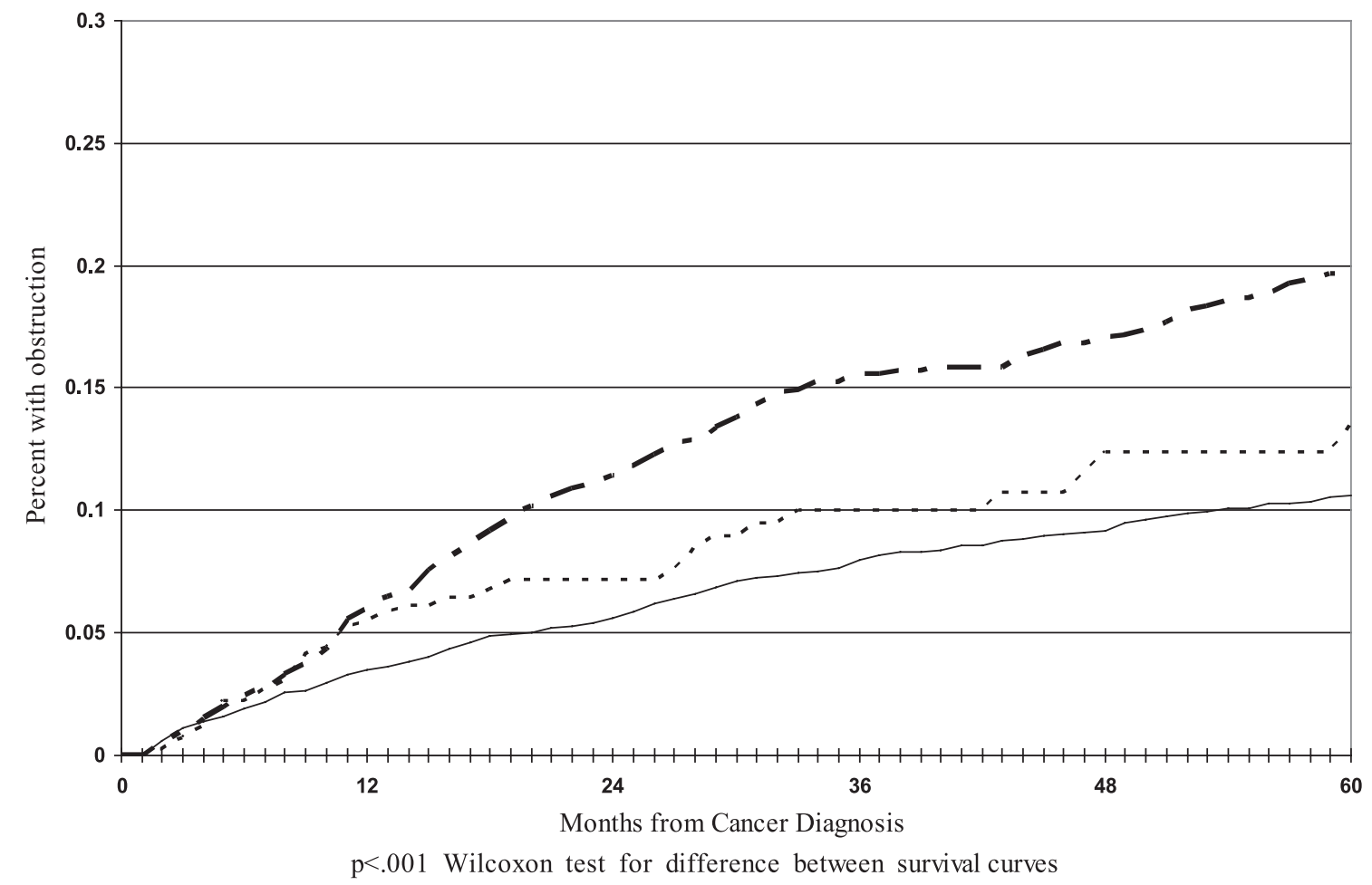

Number at Risk

Baseline

$\begin{array}{lc} & \\ & \\ \text { Post-operative group } & 1467 \\ \text { Pre-operative group } & 425 \\ \text { Nonirradiated group } & 3612\end{array}$

30 months after diagnosis

821

183

2090
60 months after

diagnosis

393

75

1152

FIGURE 2. Time from rectal cancer diagnosis to small bowel obstruction, evaluating sequence of irradiation and surgery $\left(^{*}\right)$. Irradiated group-postoperative sequence is represented by the broken line; irradiated group-preoperative sequence, dotted line; nonirradiated group, solid line. *A total of 102 patients (2.0\% of total cohort) were excluded as sequence of irradiation and surgery was unknown.

in a clinically significant increase in the cumulative incidence of SBO in patients undergoing postoperative irradiation over time. Bowel obstruction as a long term complication of irradiation has been well described; the risk increases when substantial amounts of small bowel are included in the irradiation field, particularly when doses above 50 to 55 Gy are delivered. ${ }^{8}$ Small bowel exposed to a significant dose of irradiation can, in the long term, develop fibrosis and ischemia that may manifest as SBO. However, the reported rates for SBO after pelvic irradiation vary widely and can be difficult to interpret; SBO and diarrhea as measures of longterm small bowel toxicity are often combined into a single measure of toxicity when clinical trials are reported. SBO can be a serious complication; in our study, $32 \%$ of patients developing SBO required surgery, and $25 \%$ had multiple admissions for SBO. In addition, $8.1 \%$ of patients admitted for SBO died during admission.
While demonstrating and quantifying an increased risk of SBO with irradiation, our study also found that timing of irradiation modified SBO risk. Within the first year, patients undergoing preoperative irradiation had a higher risk of SBO than those not undergoing irradiation (although this increase was not statistically significant). However, after the first year, the risk of SBO was similar between these groups and was substantially lower than in patients undergoing postoperative irradiation. Adhesions commonly form after pelvic surgery, and this may lead to fixation of small bowel loops in the pelvis. Green et $\mathrm{al}^{18,19}$ found that $65 \%$ of patients referred for postoperative irradiation had fixed loops of small bowel within the pelvis as compared with $18 \%$ of patients with no prior surgery. Bowel that is fixed in the pelvis is more likely to receive a significant cumulative dose of irradiation than mobile bowel, as fixed bowel will maintain the same position in the pelvis over the course of treatment. However, most 
TABLE 2. Proportional Hazards Model Predicting Time to Small Bowel Obstruction Including Any Irradiation*

\begin{tabular}{lcc}
\hline Variable & $\begin{array}{c}\text { Hazard Ratio } \\
\mathbf{( 9 5 \% ~ C I ) ~}\end{array}$ & $\boldsymbol{P}$ \\
\hline Radiation status & 1.0 (referent) & $<0.0001$ \\
$\quad$ Surgery only group (nonirradiated) & $1.53(1.28-1.83)$ & \\
$\quad$ Irradiated group & $0.99(0.92-1.06)$ & 0.74 \\
Age (yr) at diagnosis & & \\
$\quad$ 5-yr intervals (65-69 through 85+) & 1.0 (referent) & $<0.0001$ \\
Stage & $0.59(0.49-0.71)$ & \\
$\quad$ Regional & & \\
$\quad$ Localized & $1.51(1.04-2.18)$ & 0.03 \\
Race & 1.0 & \\
$\quad$ Black & & \\
$\quad$ Non-Black & $1.01(0.86-1.18)$ & 0.95 \\
Sex & 1.0 & \\
$\quad$ Male & & \\
$\quad$ Female & $1.02(0.87-1.20)$ & 0.78 \\
Type of surgery & 1.0 & \\
$\quad$ APR & Non-APR &
\end{tabular}

*Adjusted for registry, time period of diagnosis, and all other factors in the model.

patients treated with preoperative irradiation in our study were diagnosed after 1996; and although we controlled for time period, it is possible that the substantial improvements in irradiation technique that have occurred over time may have confounded our results.

TABLE 3. Proportional Hazards Model Predicting Time to Small Bowel Obstruction in Patients Surviving 1 Year After Diagnosis*

\begin{tabular}{|c|c|c|}
\hline Variable & $\begin{array}{c}\text { Hazard Ratio } \\
(95 \% \text { CI })\end{array}$ & $\boldsymbol{P}$ \\
\hline \multicolumn{3}{|l|}{ Irradiation status $^{\dagger}$} \\
\hline Surgery only (nonirradiated) & 1.0 (referent) & \multirow[t]{3}{*}{$<0.0001$} \\
\hline Surgery + postoperative irradiation & $1.69(1.34-2.14)$ & \\
\hline Surgery + preoperative irradiation & $0.89(0.55-1.46)$ & \\
\hline \multicolumn{3}{|l|}{ Age (yr) at diagnosis } \\
\hline 5 -yr intervals (65-69 through $85+$ ) & $0.98(0.90-1.07)$ & 0.60 \\
\hline \multicolumn{3}{|l|}{ Stage } \\
\hline Regional & 1.0 (referent) & \multirow[t]{2}{*}{$<0.0001$} \\
\hline Localized & $0.55(0.43-0.68)$ & \\
\hline \multicolumn{3}{|l|}{ Race } \\
\hline Black & $1.71(1.08-2.70)$ & \multirow[t]{2}{*}{0.02} \\
\hline Non-Black & 1.0 & \\
\hline \multicolumn{3}{|l|}{ Sex } \\
\hline Male & $1.09(0.89-1.33)$ & \multirow[t]{2}{*}{0.42} \\
\hline Female & 1.0 & \\
\hline \multicolumn{3}{|l|}{ Type of surgery } \\
\hline APR & $0.92(0.75-1.12)$ & 0.40 \\
\hline Non-APR & 1.0 & \\
\hline
\end{tabular}

Our findings are consistent with the results of recent randomized control trials of preoperative irradiation. In the Dutch trial of total mesorectal excision with and without short-course preoperative irradiation, ${ }^{3,12} 11 \%$ of patients in the surgery only group and $11 \%$ of patients in the surgery with irradiation group were admitted with a diagnosis of SBO over a median follow-up of 5.1 years, indicating no increased risk of SBO in patients treated with short course preoperative irradiation. In the German trial comparing preoperative irradiation to postoperative irradiation for advanced rectal cancer, ${ }^{7}$ long-term gastrointestinal side effects of irradiation (chronic diarrhea or obstruction as a combined outcome) occurred in $9 \%$ of patients undergoing preoperative treatment as compared with $15 \%$ of patients undergoing postoperative irradiation. Similar to these findings, the results of our study reinforce the superiority of preoperative therapy in terms of reducing the long-term risk of SBO.

Our study used cancer registry and administrative data and therefore has several limitations. We did not have information about irradiation dosage or fields, and we had no information about the use of techniques to minimize irradiation exposure of small bowel, such as positioning or the use of belly boards, and the use of such techniques may have increased over time. However, because SEER is populationbased, our patients would have received the standard care for their communities. We did not evaluate the use of chemotherapy. It is possible that the use of chemosensitization with irradiation may increase the susceptibility of small bowel to acute and/or chronic toxicity and, therefore, some of the effect of irradiation demonstrated in our study may be due to the combination of chemotherapy and irradiation. Not all patients in our study would have received chemosensitization; thus, given the current North American standard of care, ${ }^{20}$ our results may underestimate the risk of SBO with treatment in the present era. We found that stage was associated with the risk of SBO; patients with a more advanced stage of disease were more likely to develop SBO. While it is possible that the finding of more advanced cancer might alter surgical management in ways that could affect the risk of $\mathrm{SBO}$, patients who received postoperative RT were at increased risk of SBO irrespective of stage of disease.

Because our study relied on observational data, rather than on the results of a randomized trial, the potential for patient selection bias, although small, remains. For example, we did not evaluate prior surgery, a known risk factor for SBO; however, prior surgery should not have resulted in differential treatment selection. In addition, our study was restricted to the elderly, and it is possible that irradiation may have a differential effect in younger patients. However, it is unlikely that the limitations of our study would have altered our final conclusions: namely, that postoperative irradiation, but not preoperative irradiation, for rectal cancer in older patients results in an increased risk of SBO over time. Our study provides further evidence of the superiority of preoperative irradiation and supports a continued change of practice to the use of this method when adjuvant irradiation for rectal cancer is needed. 


\section{REFERENCES}

1. NIH Consensus Conference. Adjuvant therapy for patients with colon and rectal cancer. JAMA. 1990;264:1444-1450.

2. Swedish Rectal Cancer Trial. Improved survival with preoperative radiotherapy in resectable rectal cancer. N Engl J Med. 1997;336:980-987.

3. Kapiteijn E, Marijnen CA, Nagtegaal ID, et al. Dutch Colorectal Cancer Group: preoperative radiotherapy combined with total mesorectal excision for resectable rectal cancer. $N$ Engl J Med. 2001;345:638-646.

4. Colorectal Cancer Collaborative Group. Adjuvant radiotherapy for rectal cancer: a systematic overview of 8,507 patients from 22 randomised trials. Lancet. 2001;358:1291-1304.

5. Wolmark N, Wieand HS, Hyams DM, et al. Randomized trial of postoperative adjuvant chemotherapy with or without radiotherapy for carcinoma of the rectum: National Surgical Adjuvant Breast and Bowel Project Protocol R-02. J Natl Cancer Inst. 2000;92:388-396.

6. Baxter NN, Rothenberger DA, Morris AM, et al. Adjuvant radiation for rectal cancer: do we measure up to the standard of care? An epidemiologic analysis of trends over 25 years in the United States. Dis Colon Rectum. 2005;48:9-15.

7. Sauer R, Becker H, Hohenberger W, et al. German Rectal Cancer Study Group: preoperative versus postoperative chemoradiotherapy for rectal cancer. N Engl J Med. 2004;351:1731-1740.

8. Coia LR, Myerson RJ, Tepper JE. Late effects of radiation therapy on the gastrointestinal tract. Int J Radiat Oncol Biol Phys. 1995;31:1213-1236.

9. Andreyev J. Gastrointestinal complications of pelvic radiotherapy: are they of any importance? Gut. 2005;54:1051-1054.

10. Miller AR, Martenson JA, Nelson H, et al. The incidence and clinical consequences of treatment-related bowel injury. Int J Radiat Oncol Biol Phys. 1999;43:817-825.
11. Ellis H, Moran BJ, Thompson JN, et al. Adhesion-related hospital readmissions after abdominal and pelvic surgery: a retrospective cohort study. Lancet. 1999;353:1476-1480.

12. Peeters KC, van de Velde CJ, Leer JW, et al. Late side effects of short-course preoperative radiotherapy combined with total mesorectal excision for rectal cancer: increased bowel dysfunction in irradiated patients. A Dutch colorectal cancer group study. J Clin Oncol. 2005;23: 6199-6206.

13. Surveillance, Epidemiology and End Results Program Website. Public Use Data. http://seer.cancer.gov/publicdata/. Accessed April 23, 2006.

14. Potosky AL, Riley GF, Lubitz JD, et al. Potential for cancer related health services research using a Medicare-tumor registry database. Med Care. 1993;31:732-748.

15. U.S. Public Health Service. International Classification of Diseases, 9th revision [DHHS Publication No. (PHS) 92-1260]. Washington, DC: U.S. GPO, 1992.

16. Baxter NN, Habermann EB, Tepper JE, et al. Risk of pelvic fractures in older women following pelvic irradiation. JAMA. 2005;294:2587-2593.

17. Parker MC, Wilson MS, Menzies D, et al. Surgical and Clinical Adhesions Research (SCAR) Group. Colorectal surgery: the risk and burden of adhesion-related complications. Colorectal Dis. 2004;6:506-511.

18. Green N. The avoidance of small intestine injury in gynecologic cancer. Int J Radiat Oncol Biol Phys. 1983;9:1385-1390.

19. Green N, Iba G, Smith WR. Measures to minimize small intestine injury in the irradiated pelvis. Cancer. 1975;35:1633-1640.

20. Engstrom PF, Benson AB 3rd, Chen YJ, et al. National Comprehensive Cancer Network: rectal cancer clinical practice guidelines in oncology. J Natl Compr Canc Netw. 2005;3:492-508. 